\section{Distúrbio de voz e estresse no trabalho docente: um estudo caso-controle}

\author{
Voice disorders related to job stress in teaching: \\ a case-control study
}

\begin{abstract}
This case-control study aimed to test the association between voice disorders and job stress among public schoolteachers in São Paulo, Brazil. The groups consisted of teachers with $(n=165)$ and without $(n=105)$ voice-related complaints. Both groups answered the questionnaires Conditions of Vocal Production and Job Stress Scale. Analysis of cases and controls showed comparable samples, differing only by vocal symptoms. There was a statistically significant difference between cases and controls in relation to job stress involving high strain (OR = 2.1; 95\%CI: 1.1-3.9), which places high demands combined with low job control. High strain in cases in this study represents the highest risk of physical and mental disorders for teachers. Loss of voice prevents teachers from continuing in their professional role, eliminates their professional identity, and jeopardizes their career.
\end{abstract}

Voice Disorders; Faculty; Ocupational Health
Susana Pimentel Pinto Giannini 1,2 Maria do Rosário Dias de Oliveira Latorre 1 Leslie Piccolotto Ferreira 2

\section{Introdução}

O desenvolvimento do distúrbio vocal decorrente do uso profissional da voz tem se mostrado, cada vez mais, associado ao trabalho docente e levado os professores a situações de afastamento e incapacidade para o desempenho de suas funções, o que implica custos financeiros e sociais 1 .

Estudos indicam consistente associação entre o ambiente escolar e a ocorrência do distúrbio de voz entre os educadores, especialmente em escolas infantis e fundamentais 2 . Ruído excessivo, limpeza insatisfatória, iluminação e tamanho da sala inapropriados, entre outros, são fatores associados ao distúrbio de voz do professor 3,4,5,6.

Características pessoais, como hábito de falar muito ou gritar, e aspectos biológicos, como a presença de alergia ou refluxo faringolaríngeo, associados às características ambientais impróprias da escola favorecem, mas não são causas suficientes para a ocorrência do distúrbio de voz. Aspectos do ambiente físico, químico e biológico afetam psiquicamente o trabalhador, principalmente se intensificados por tempo de exposição ou ritmo da organização do trabalho ${ }^{7}$. Assim, o trabalho em ambiente ruidoso, com temperatura elevada ou em contato com substâncias químicas irritativas demanda maior esforço para concentração de atenção e, portanto, quanto maior a jornada, maior o desgaste. A intensificação de esforços como forma de lidar com a sobrecarga no trabalho pode ser de ordem física, cognitiva ou 
afetiva ${ }^{7}$. Há de se considerar, portanto, os aspectos que têm origem nas formas de organização do trabalho docente, referentes ao conteúdo e à divisão do trabalho e às relações interpessoais ${ }^{8}$.

Como variáveis indicadoras de estresse associadas ao distúrbio de voz de professores, pesquisas indicam experiências de violência na escola, dificuldades de relacionamento no trabalho, baixa autonomia, pouca possibilidade de criatividade nas atividades, falta de tempo para correção de tarefas e provas, além das más condições de trabalho em geral e das mudanças político-educacionais constantes 9 . Tais características do trabalho docente podem favorecer o adoecimento físico ou psíquico do professor.

Entretanto, ainda que estudos apontem essa associação, não é possível estabelecer relação causal entre o adoecimento vocal e o trabalho docente, porque, assim como outras doenças funcionais contemporâneas, o distúrbio de voz tem, por característica, a causalidade difusa e complexa, não objetiva e linear.

Considerando o desafio de avaliar os efeitos do trabalho na saúde desses trabalhadores e das diversas propostas teóricas e metodológicas elaboradas na perspectiva de apresentar modelos para estudar a dimensão do adoecimento relacionado ao trabalho 10 , o presente estudo utiliza o modelo demanda-controle 11 , que tem foco no modo de organização do trabalho e busca identificar a forma como o trabalhador vivencia seu contexto de trabalho.

O objetivo deste estudo é determinar a associação entre o distúrbio de voz e o estresse no trabalho docente entre professoras da rede municipal de São Paulo, Brasil.

\section{Método}

O estudo caso-controle foi realizado com professoras do ensino infantil, fundamental e médio da rede de educação do Município de São Paulo. Foram excluídas professoras que apresentaram alterações em pregas vocais não associadas ao uso da voz e que estavam afastadas da sala de aula por licença médica ou readaptação de função.

A seleção de participantes constou de duas etapas. Da primeira etapa, participaram todas as professoras que compareceram ao Setor de Fonoaudiologia do Hospital do Servidor Público Municipal de São Paulo com queixa de alteração vocal no período de julho/2007 a maio/2009. As educadoras foram submetidas à avaliação vocal, laringoscópica e responderam a questionários. $\mathrm{Na}$ segunda etapa, as pesquisadoras foram às escolas das professoras participantes do estudo e sortearam professoras sem queixa de voz que estavam em atividade em sala de aula. Essas educadoras selecionadas foram submetidas aos mesmos procedimentos da etapa anterior, e, caso alguma professora apresentasse distúrbio de voz, era classificada no grupo de caso; caso a professora fosse classificada sem distúrbio de voz, era alocada no grupo de controle.

Para avaliação de voz, as coletas de amostra de fala foram realizadas por fonoaudiólogas, sempre no mesmo dia da semana, às sextas-feiras, no início da manhã, a fim de garantir descanso vocal mínimo (noturno). O registro foi realizado diretamente em computador portátil, com uso de microfone de cabeça. Optou-se pela análise perceptivo-auditiva, por ser um procedimento soberano na avaliação da qualidade vocal, e pela escala GRBASI (Grade, Roughness, Breathiness, Asteny, Strain, Instability) 12,13, por ser um instrumento de uso internacional e de alto grau de confiabilidade. A avaliação das vozes foi realizada por análise simultânea de três juízas, fonoaudiólogas especialistas e com experiência na área. As juízas não participaram da coleta de amostra de fala e não tinham conhecimento da identificação dos sujeitos. A voz foi classificada em com alteração, quando a alteração foi julgada moderada (grau 2) ou intensa (grau 3), e sem alteração, quando normal (grau 0) ou leve (grau 1).

Todas as avaliações otorrinolaringológicas foram efetuadas pelo mesmo médico, otorrinolaringologista e foniatra com experiência clínica em laringologia, sempre às sextas-feiras, após a coleta de amostra de fala. Foi realizada videolaringoscopia com laringoscópio rígido e flexível sob anestesia local (spray de lidocaína), quando necessária. $O$ protocolo de avaliação incluiu aspectos otorrinolaringológicos gerais e laríngeos específicos. Os sujeitos foram classificados em com alteração, na presença de lesão, alteração irritativa, estrutural ou de coaptação de pregas vocais, ou sem alteração, na ausência de qualquer lesão ou alteração visível.

No presente estudo, a definição de caso foi baseada nos resultados das avaliações vocal e laringoscópica. Como caso, foram classificadas as professoras que apresentaram alteração na avaliação vocal (GRBASI 2 ou 3) e presença de lesão ou alteração irritativa, estrutural ou de coaptação de pregas vocais em avaliação otorrinolaringológica perceptivo-visual. Como controle, foram consideradas as participantes com ausência ou alteração leve de qualidade vocal na avaliação perceptivo-auditiva (GRBASI 0 e 1), bem como ausência de alteração na avaliação otorrinolaringológica. Foram excluídas da amostra todas as professoras que apresentaram alteração em apenas uma das avaliações, com o objetivo de compor grupos bem distintos pela doença em 
questão, ainda que as mesmas tenham sido consideradas casos de distúrbio de voz do ponto de vista clínico e acolhidas para tratamento.

No cálculo do tamanho da amostra, foi assumido erro tipo I de $5 \%$, poder de teste de $80 \%$, frequência esperada máxima de exposição entre os controles de $40 \%$, valor mínimo de odds ratio (OR) de 2,5 e estimou-se que seriam necessários 85 casos e 85 controles.

Foram utilizados dois questionários: (1) Condição de Produção Vocal - Professor (CPV-P), instrumento utilizado em diversas pesquisas no Brasil, é adequado para caracterizar as condições de ambiente escolar e perfil vocal de professores. Neste estudo, as respostas forneceram os dados das variáveis sociodemográficas, de estilo de vida, de ocupação e de ambiente e organização do trabalho docente 14; (2) Job Stress Scale (JSS), instrumento que avalia as dimensões de demanda, controle e apoio no trabalho referentes às fontes de estresse no ambiente psicossocial do trabalho e o desgaste resultante de sua interação. Tem origem nos estudos de Karasek 15 sobre a repercussão do estresse proveniente da organização do trabalho na saúde. Foi utilizada a versão resumida adaptada para o português 11 , com 17 questões em três dimensões: demanda, controle e apoio. Demanda é qualquer tipo de pressão de natureza psíquica para realização de um trabalho, que pode ser tanto quantitativa, como pressão de velocidade e tempo, como qualitativa, referente à execução de tarefas contraditórias, por exemplo. Controle é a possibilidade que o trabalhador tem de utilizar as habilidades intelectuais para realizar seu trabalho e a autoridade que possui para tomar decisões. A terceira dimensão refere-se ao apoio social no ambiente de trabalho, sendo que a falta dessa interação social pode gerar consequências negativas à saúde do trabalhador 11. Neste estudo, a análise foi realizada considerando os quadrantes resultantes das interações entre as dimensões, a saber: alto controle e baixa demanda (baixa exigência), alto controle e alta demanda (trabalho ativo), alto controle e baixa demanda (trabalho passivo) e baixo controle e alta demanda (alto desgaste). As condições de baixo desgaste e de trabalho ativo são consideradas ideal e boa, respectivamente, uma vez que favorecem a criatividade e podem motivar o desenvolvimento de novos comportamentos. As condições de trabalho passivo e alto desgaste são as mais nocivas, sendo que a segunda, alto desgaste, é a que apresenta maiores riscos de exigência psicológica e adoecimento psíquico ao trabalhador 11 .

A variável dependente foi a presença de distúrbio de voz ( $\operatorname{sim}$ = caso; não = controle); a variável independente de interesse foi o estresse no trabalho. Como variáveis independentes de controle, foram consideradas as características sociodemográficas (idade, estado civil, escolaridade), de estilo de vida (tabagismo, etilismo), da função docente (tempo de profissão, vínculo como professora, horas/aulas por semana), do ambiente (presença de ruído, de eco, de poeira, de umidade, temperatura agradável, adequação da acústica, do tamanho da sala, da iluminação, da limpeza da escola e dos banheiros, utilização de produtos químicos irritativos na limpeza) e da organização do trabalho (se o ambiente é calmo, se há supervisão constante, se o ritmo é estressante, se há tempo para realizar todas as atividades na escola, se há local de descanso, se há facilidade para sair da sala, se tem satisfação na função, se há comprometimento dos funcionários com a manutenção da escola, se o trabalho é monótono, se o trabalho é repetitivo, se há estresse no trabalho, se há situações de violência e com qual frequência: depredação, roubo de objetos pessoais, ameaça ao professor, necessidade de intervenção da polícia, manifestação de racismo, indisciplina, briga, agressão, insulto, violência à porta da escola, violência contra funcionários, problemas com drogas e pichação).

Na análise estatística, avaliou-se, inicialmente, a consistência interna do JSS por meio do cálculo do coeficiente alfa de Cronbach. A seguir, foram realizadas as análises descritiva e de associação com a variável de interesse para comparação dos grupos de caso e de controle pelo teste de associação pelo qui-quadrado, com correção de Yates. Foram estimados modelos de regressão logística para calcular a razão de chances (OR) bruta e ajustada, com respectivos intervalos de 95\% de confiança (IC95\%), para avaliar os riscos em relação à variável independente de interesse. A avaliação do ajuste da análise de regressão logística foi feita pelo teste de Hosmer-Lemeshow.

A pesquisa foi aprovada pelos Comitês de Ética em Pesquisa da Faculdade de Saúde Pública, Universidade de São Paulo (USP - no. 173/07) e do Hospital do Servidor Público Municipal ( $\mathrm{n}^{\circ}$. 101/07). Todas as participantes receberam esclarecimentos e concordaram em participar do estudo ao assinar o Termo de Consentimento Livre e Esclarecido. Este estudo contou com o apoio financeiro da Fundação de Amparo à Pesquisa do Estado de São Paulo (FAPESP).

\section{Resultados}

Foram realizadas 354 avaliações; e, ao final, o grupo de caso contou com 167 sujeitos, e o grupo de controle, com 105. A análise dos grupos revela que as amostras são comparáveis, uma vez que 
não há diferença estatisticamente significativa em relação às características sociodemográficas e de estilo de vida, conforme se observa na Tabela 1. Como a idade mostrou valor de $p<0,10$, foi selecionada como variável de controle na análise de regressão logística.

Também não se observa diferença estatística entre os grupos de caso e de controle na caracterização das condições do ambiente (Tabela 2) e da organização do trabalho (Tabela 3), situação esperada uma vez que os grupos eram seleciona- dos nos mesmos locais de trabalho A única variável que apresenta diferença estatística é acústica insatisfatória $(\mathrm{p}=0,010)$, também adicionada na análise de regressão logística para ajuste.

Em relação aos sintomas vocais e sensações laringofaríngeas, os grupos se diferenciam, visto que todos os sintomas avaliados têm associação estatisticamente significativa na distinção dos grupos. No grupo de caso, há maior presença de rouquidão $(93,4 \%$ casos; $51 \%$ controles; $\mathrm{p}<0,001)$, de episódios de perda de voz $(57,6 \%$

\begin{tabular}{|c|c|c|c|c|c|}
\hline \multirow[t]{2}{*}{ Características sociodemográficas e de estilo de vida } & \multicolumn{2}{|c|}{ Controles $(n=105)$} & \multicolumn{2}{|c|}{ Casos $(n=167)$} & \multirow[t]{2}{*}{ Valor de $p\left(\chi^{2}\right)$} \\
\hline & $\mathbf{n}$ & $\%$ & n & $\%$ & \\
\hline \multicolumn{6}{|l|}{ Idade (anos) } \\
\hline $20-29$ & 15 & 14,3 & 21 & 12,6 & 0,092 \\
\hline $30-39$ & 38 & 36,2 & 50 & 29,9 & \\
\hline $40-49$ & 33 & 31,4 & 77 & 46,1 & \\
\hline $50-65$ & 19 & 18,1 & 19 & 11,4 & \\
\hline \multicolumn{6}{|l|}{ Estado civil } \\
\hline Solteira & 27 & 25,7 & 49 & 29,3 & 0,513 \\
\hline Casada & 62 & 59,0 & 100 & 59,9 & \\
\hline Separada/Viúva & 16 & 15,2 & 18 & 10,8 & \\
\hline \multicolumn{6}{|l|}{ Escolaridade } \\
\hline Até superior incompleto & 4 & 3,8 & 13 & 7,8 & 0,187 \\
\hline Superior completo e mais & 101 & 96,2 & 154 & 92,2 & \\
\hline \multicolumn{6}{|l|}{ Tempo de profissão (anos) } \\
\hline$\leq 10$ & 33 & 31,7 & 40 & 24 & 0,244 \\
\hline $11-15$ & 23 & 22,1 & 29 & 17,4 & \\
\hline $16-20$ & 29 & 27.9 & 62 & 37,1 & \\
\hline$\geq 21$ & 19 & 18,3 & 36 & 21,5 & \\
\hline \multicolumn{6}{|l|}{ Vínculo } \\
\hline Professora titular & 101 & 96,2 & 158 & 94,6 & 0,552 \\
\hline Professora substituta & 4 & 3,8 & 9 & 5,4 & \\
\hline \multicolumn{6}{|l|}{ Aulas/semana (horas) } \\
\hline$\leq 10$ & 14 & 13,3 & 29 & 17,4 & 0,187 \\
\hline $11-20$ & 16 & 15,2 & 22 & 13,2 & \\
\hline $21-30$ & 32 & 30,5 & 31 & 18,6 & \\
\hline $31-40$ & 24 & 22,9 & 48 & 28,7 & \\
\hline$\geq 41$ & 19 & 18,1 & 37 & 22,2 & \\
\hline \multicolumn{6}{|l|}{ Tabagismo } \\
\hline Não fumante & 84 & 74,1 & 132 & 79,0 & 0,238 \\
\hline Ex-fumante & 11 & 10,5 & 16 & 9,6 & \\
\hline Fumante & 10 & 9,5 & 19 & 11,4 & \\
\hline \multicolumn{6}{|l|}{ Etilismo } \\
\hline Nunca & 45 & 43,3 & 78 & 46,7 & 0,666 \\
\hline Raramente & 43 & 41,3 & 60 & 35,9 & \\
\hline Às vezes & 16 & 15,4 & 29 & 17,4 & \\
\hline
\end{tabular}


Tabela 2

Distribuição de casos e controles, segundo características do ambiente físico do trabalho.

\begin{tabular}{|c|c|c|c|c|c|}
\hline \multirow[t]{2}{*}{ Aspectos do ambiente físico do trabalho } & \multicolumn{2}{|c|}{ Controles $(n=105)$} & \multicolumn{2}{|c|}{ Casos $(n=167)$} & \multirow[t]{2}{*}{ Valor de $p\left(\chi^{2}\right)$} \\
\hline & $\mathrm{n}$ & $\%$ & $\mathrm{n}$ & $\%$ & \\
\hline \multicolumn{6}{|l|}{ Presença de ruído } \\
\hline Não & 3 & 2,9 & 4 & 2,4 & 0,791 \\
\hline Sim & 99 & 97,1 & 162 & 97,6 & \\
\hline \multicolumn{6}{|l|}{ Acústica satisfatória } \\
\hline Não & 20 & 19,0 & 55 & 33,5 & 0,010 \\
\hline Sim & 85 & 81,0 & 109 & 66,5 & \\
\hline \multicolumn{6}{|l|}{ Presença de eco } \\
\hline Não & 19 & 18,3 & 35 & 21,3 & 0,541 \\
\hline Sim & 85 & 81,7 & 129 & 78,7 & \\
\hline \multicolumn{6}{|l|}{ Presença de poeira } \\
\hline Não & 10 & 9,6 & 1 & 7,2 & 0,476 \\
\hline Sim & 94 & 90,4 & 155 & 92,8 & \\
\hline \multicolumn{6}{|l|}{ Presença de umidade } \\
\hline Não & 24 & 23,5 & 39 & 24,1 & 0,919 \\
\hline Sim & 78 & 76,5 & 123 & 75,9 & \\
\hline \multicolumn{6}{|l|}{ Temperatura agradável } \\
\hline Não & 22 & 21,6 & 41 & 24,7 & 0,557 \\
\hline Sim & 80 & 78,4 & 125 & 75,3 & \\
\hline \multicolumn{6}{|l|}{ Tamanho da sala adequado } \\
\hline Não & 53 & 51,0 & 91 & 54,5 & 0,571 \\
\hline $\operatorname{Sim}$ & 51 & 49,0 & 76 & 45,5 & \\
\hline \multicolumn{6}{|l|}{ Iluminação adequada } \\
\hline Não & 7 & 6,7 & 9 & 9 & 0,663 \\
\hline Sim & 98 & 93,3 & 94,6 & 158 & \\
\hline \multicolumn{6}{|l|}{ Limpeza satisfatória na escola } \\
\hline Não & 16 & 15,2 & 21 & 12,6 & 0,533 \\
\hline $\operatorname{Sim}$ & 89 & 84,8 & 146 & 87,4 & \\
\hline \multicolumn{6}{|l|}{ Limpeza satisfatória nos banheiros } \\
\hline Não & 6 & 5,7 & 20 & 12,0 & 0,087 \\
\hline Sim & 99 & 94,3 & 147 & 88,0 & \\
\hline \multicolumn{6}{|l|}{ Produtos de limpeza causam irritação } \\
\hline Não & 24 & 22,9 & 31 & 18,7 & 0,404 \\
\hline Sim & 81 & 77,1 & 135 & 81,3 & \\
\hline
\end{tabular}

casos; $20,4 \%$ controles; $\mathrm{p}<0,001$ ), de cansaço ao falar $(86,7 \%$ casos; $50,0 \%$ controles; $\mathrm{p}<0,001)$ e de esforço ao falar $(86,1 \%$ casos; $52,4 \%$ controles; $p<0,001$ ), situação esperada uma vez que os grupos foram compostos para se diferenciar pela doença em questão.

A Tabela 4 apresenta a análise de associação das dimensões do estresse no trabalho (JSS) com os grupos de caso e de controle e o modelo de regressão logística, ajustado por idade e acústica.

\section{Discussão}

Este estudo caso-controle avaliou a associação entre a presença de distúrbio de voz em professoras da rede municipal de ensino de São Paulo e o estresse no trabalho docente. Foi encontrada diferença estatisticamente significativa entre $o$ grupo de caso e de controle na condição de alto desgaste $(\mathrm{OR}=2,1$; IC95\%: 1,1-3,9). A categoria de alto desgaste representa alta demanda associada a baixo controle do trabalho, situação na qual se encontra a maioria das reações adversas 
Tabela 3

Distribuição de casos e controles, segundo características da organização do trabalho.

\begin{tabular}{|c|c|c|c|c|c|}
\hline \multirow[t]{2}{*}{ Aspectos da organização do trabalho } & \multicolumn{2}{|c|}{ Controles $(n=105)$} & \multicolumn{2}{|c|}{ Casos $(n=167)$} & \multirow[t]{2}{*}{ Valor $p\left(\chi^{2}\right)$} \\
\hline & $\mathbf{n}$ & $\%$ & $\mathrm{n}$ & $\%$ & \\
\hline \multicolumn{6}{|l|}{ Ambiente calmo } \\
\hline Não & 37 & 35,6 & 60 & 37,5 & 0,751 \\
\hline Sim & 67 & 64,4 & 100 & 62,5 & \\
\hline \multicolumn{6}{|l|}{ Supervisão constante } \\
\hline Não & 11 & 13,2 & 22 & 13,2 & 0,582 \\
\hline Sim & 90 & 89,1 & 145 & 86,8 & \\
\hline \multicolumn{6}{|l|}{ Ritmo estressante } \\
\hline Não & 1 & 1,0 & 2 & 1,2 & 0,843 \\
\hline Sim & 104 & 99,0 & 163 & 98,8 & \\
\hline \multicolumn{6}{|l|}{ Tempo para realizar as atividades } \\
\hline Não & 19 & 18,1 & 37 & 22,6 & 0,379 \\
\hline Sim & 86 & 81,9 & 127 & 77,4 & \\
\hline \multicolumn{6}{|l|}{ Facilidade para ausentar-se da sala } \\
\hline Não & 32 & 30,5 & 68 & 41,0 & 0,081 \\
\hline Sim & 73 & 69,5 & 41 & 59,0 & \\
\hline \multicolumn{6}{|l|}{ Satisfação no exercício da função } \\
\hline Não & 4 & 3,8 & 6 & 3,6 & 0,934 \\
\hline Sim & 101 & 96,2 & 160 & 96,4 & \\
\hline \multicolumn{6}{|l|}{ Trabalho monótono } \\
\hline Não & 26 & 27,4 & 41 & 25,9 & 0,804 \\
\hline Sim & 69 & 72,6 & 117 & 74,1 & \\
\hline \multicolumn{6}{|l|}{ Trabalho repetitivo } \\
\hline Não & 19 & 18,4 & 32 & 19,5 & 0,829 \\
\hline Sim & 84 & 81,6 & 132 & 80,5 & \\
\hline \multicolumn{6}{|l|}{ Presença de violência/Depredação } \\
\hline Não & 23 & 22,1 & 42 & 25,3 & 0,551 \\
\hline Sim & 81 & 77,9 & 124 & 74,7 & \\
\hline \multicolumn{6}{|l|}{ Roubo de objetos pessoais } \\
\hline Não & 36 & 34,6 & 60 & 35,9 & 0,826 \\
\hline Sim & 68 & 65,4 & 107 & 64,1 & \\
\hline \multicolumn{6}{|l|}{ Ameaça ao professor } \\
\hline Não & 28 & 26,9 & 51 & 30,5 & 0,524 \\
\hline Sim & 76 & 73,1 & 116 & 69,5 & \\
\hline \multicolumn{6}{|l|}{ Manifestação de racismo } \\
\hline Não & 31 & 30,1 & 56 & 33,5 & 0,557 \\
\hline Sim & 72 & 69,9 & 111 & 66,5 & \\
\hline \multicolumn{6}{|l|}{ Indisciplina } \\
\hline Não & 7 & 6,7 & 10 & 6,0 & 0,832 \\
\hline Sim & 98 & 93,3 & 156 & 94,0 & \\
\hline \multicolumn{6}{|l|}{ Brigas } \\
\hline Não & 18 & 17,1 & 28 & 17,0 & 0,971 \\
\hline Sim & 87 & 82,9 & 137 & 83,0 & \\
\hline \multicolumn{6}{|l|}{ Violência contra funcionários } \\
\hline Não & 34 & 32,4 & 52 & 31,3 & 0,856 \\
\hline Sim & 71 & 67,6 & 114 & 68,7 & \\
\hline \multicolumn{6}{|l|}{ Problemas com drogas } \\
\hline Não & 29 & 27,6 & 38 & 22,8 & 0,365 \\
\hline Sim & 76 & 72,4 & 129 & 77,2 & \\
\hline
\end{tabular}


Análise de associação e análise de regressão logística do escore do Job Stress Scale (JSS) com os grupos de caso e de controle.

\begin{tabular}{|c|c|c|c|c|c|c|c|}
\hline \multirow[t]{2}{*}{$\begin{array}{l}\text { JSS modelo controle/ } \\
\text { demanda }\end{array}$} & \multicolumn{2}{|c|}{$\begin{array}{l}\text { Controles } \\
(n=105)\end{array}$} & \multicolumn{2}{|c|}{$\begin{array}{c}\text { Casos } \\
(n=167)\end{array}$} & \multirow{2}{*}{$\begin{array}{c}\text { Valor } \\
\text { de } p \\
\chi^{2}\end{array}$} & \multicolumn{2}{|c|}{$\begin{array}{l}\text { Modelo de } \\
\text { regressão logística }\end{array}$} \\
\hline & $n$ & $\%$ & $\mathrm{n}$ & $\%$ & & OR (valor de p) & IC95\% \\
\hline Baixa exigência & 30 & 29,7 & 30 & 18,3 & 0,171 & 1,0 & \\
\hline Trabalho ativo & 8 & 7,9 & 14 & 8,5 & & $1,8(0,275)$ & $0,6-4,8$ \\
\hline Trabalho passivo & 27 & 26,7 & 46 & 28,0 & & $1,7(0,132)$ & $0,9-3,4$ \\
\hline Alto desgaste & 36 & 35,6 & 74 & 45,1 & & $2,1(0,028)$ & $1,1-3,9$ \\
\hline
\end{tabular}

IC95\%: intervalo de 95\% de confiança; OR: odds ratio, ajustado por idade e acústica.

das exigências psicológicas, tais como fadiga, ansiedade, depressão e doença física 10.

Toda situação em que há baixo controle do trabalho pode produzir algum efeito na saúde advindo de perda de habilidade e desinteresse. A relação entre grande demanda e baixo controle, entretanto, gera alto desgaste e é a mais nociva ao trabalhador 16 . O trabalho em alta exigência produz situações de maior repercussão negativa sobre a saúde psíquica 10 , estando estatisticamente associado à insatisfação no trabalho, ao estresse psicológico, ou Burnout, síndrome descrita como aquela na qual o trabalhador perde o sentido de sua relação com o trabalho de forma que qualquer esforço lhe parece inútil 17 .

Não foram encontrados, na literatura consultada, estudos que utilizem o JSS associado ao distúrbio de voz do professor. Estudo realizado na Bahia 18 utilizou a escala Job Content Questionnaire (JCQ, versão ampliada, com 49 questões, da qual a JSS se originou), porém, nenhum dos termos avaliados permaneceu no modelo final obtido para os dois desfechos estudados (rouquidão e calos nas cordas vocais). Outro estudo 19 utilizou a JCQ ao analisar os fatores psicossociais do trabalho docente e encontrou associação do distúrbio de voz ao trabalho em alta exigência, caracterizado por alta demanda e baixo controle, resultado coincidente com o deste estudo. Também com a JCQ, estudo 20 avaliou a associação entre controle sobre o trabalho, demandas psicológicas e ocorrência de distúrbios psíquicos menores entre professores do ensino fundamental e concluiu que as prevalências de distúrbios psíquicos menores foram mais elevadas em professores com trabalho em alta exigência, caracterizado por alta demanda e baixo controle ( $\mathrm{RP}=1,74$; IC95\%: 1,44-2,10), e naqueles em trabalho ativo, com alta demanda e alto controle (RP = 1,35; IC95\%: 1,13-1,61), quan- do comparadas às prevalências dos professores em trabalho de baixa exigência (baixa demanda e alto controle).

Como aumento de demanda, destacam-se as recentes reformas nos sistemas de ensino que buscaram ampliar o atendimento educacional, estendendo-o aos que não têm acesso e aos que não puderam manter-se na escola ${ }^{9}$. Tais reformas geraram uma transformação nos aspectos físicos e organizacionais dos sistemas educativos, acompanhada pela adoção de critérios de eficácia, produtividade e excelência. Essa situação provoca maior demanda de atendimento, com ampliação no número de matrículas, maior número de turmas e de alunos por sala de aula, e configura uma intensificação tanto em termos qualitativos, caracterizados pelas transformações da atividade sob pressão temporal, quanto em termos quantitativos, relacionados ao aumento do volume de tarefas 9 .

Ao mesmo tempo em que aumentam a pressão e o volume da tarefa, o professor perde, progressivamente, o controle sobre as atividades docentes, múltiplas e complexas dentro da sala de aula, com simultaneidade de eventos, imprevisibilidade e imediatismo 21. É comum ao professor executar outras tarefas, como atender ao aluno individualmente, controlar a turma e preencher instrumentos e formulários, enquanto leciona, sobreposição essa de tarefas que explica o cansaço físico, vocal e mental do docente 9 .

O modelo de avaliação do estresse no trabalho adotado prediz que o trabalho na condição de alto desgaste pode conduzir ao declínio na atividade global do indivíduo e à redução da capacidade de produzir soluções para as atividades e problemas enfrentados 11. Trabalhar sob pressão desfavorece o desenvolvimento de estratégias de autoproteção à saúde, como a busca de melhor postura corporal ou projeção vocal, e resulta na 
adoção de estratégias com hipersolicitação do corpo e consequente fadiga física e mental ${ }^{9}$.

A somatória de exigências leva a um aumento de esforços que ultrapassa os limiares adequados às condições de saúde do trabalhador. Frente ao acúmulo de trabalho, o trabalhador busca, em seu repertório, novos recursos para enfrentar a situação que se apresenta 7. Essa autoexigência acarreta esforço no sentido de controlar e não demonstrar cansaço, irritação, raiva. Quanto maior o cansaço, menor a participação social e em atividades de lazer, o que contribui para que o quadro se agrave continuamente.

A ênfase nas habilidades e competências individuais do docente e suas repercussões sobre os projetos educacionais encobrem as condições de trabalho existentes: "o mal-estar docente pode ser explicado pela presença de obstáculos relacionados ao volume de trabalho e à precariedade das condições existentes, mas também às altas demandas no trabalho, incluindo as demandas emocionais, junto a uma expectativa social de excelência, cujo limite é exigir do professor uma atuação capaz de reverter a situação na qual se encontra" 22 (p. 5).

Essa dinâmica de forças contrárias conduz os professores ao adoecimento e, muitas vezes, à impossibilidade de manter-se na função docente. A decepção com a realidade encontrada é causa primeira da frustração com o trabalho docente 17,23,24. Ao abandonar a imagem idealizada e subordinar-se ao trabalho proposto, o professor elimina, em última instância, o próprio sentido do seu trabalho 25 . Ao perder a voz, o professor perde a possibilidade de manter-se em sua função, perde sua identidade profissional, o que coloca, em risco, a sua carreira e a sua sobrevivência como educador 26.

\section{Considerações metodológicas e limites do estudo}

Optou-se por esse delineamento por ser um tipo de estudo apropriado para estimar a magnitude da associação entre exposição e doença e por ser eficiente para avaliar agravos relacionados ao trabalho. A principal dificuldade metodológica, neste estudo, foi conceituar caso 27 , definição de crucial importância em estudos caso-controle. A variável dependente - distúrbio de voz - é uma manifestação dinâmica e funcional, o que impossibilita uma definição dicotômica entre doença e não doença ${ }^{28}$. Não há consenso sobre o procedimento de avaliação padrão-ouro para essa definição. No presente estudo, optou-se por definir caso pela presença de sintoma vocal atual associada à avaliação perceptivo-auditiva da voz, realizada por fonoaudiólogo, e à avaliação perceptivo-visual das pregas vocais, realizada por médico otorrinolaringologista.

Da mesma forma, a seleção de sujeitos para compor os grupos de caso e de controle mostrouse complexa. Para reduzir possível viés de seleção, optou-se por compor o grupo de controle por professoras que lecionavam nas mesmas escolas, garantindo máxima semelhança com o grupo de caso, com mesma probabilidade de exposição aos fatores de risco físicos, químicos e biológicos do ambiente de trabalho escolar.

Considerar, como critério de exclusão, o afastamento da sala de aula por licença médica ou readaptação funcional por qualquer agravo de saúde pode ter favorecido o viés do trabalhador sadio. Entretanto, a manutenção dessa parcela da população causaria distorções nas análises de comparação dos grupos, uma vez que a necessidade de uso vocal é distinta e variada nessas ocupações. Optou-se, ainda, por não considerar critério de exclusão o fato de as professoras terem recebido orientação ou tratamento, uma vez que seria impossível garantir a ausência de qualquer informação prévia, especialmente pela vigência do Programa de Saúde Vocal nas escolas da Prefeitura do Município de São Paulo 29.

Finalmente, considera-se que o delineamento de caso-controle não permite estabelecer relação causal entre a exposição e o efeito na saúde, entretanto, o estudo avança ao confirmar a associação dos aspectos de estresse no trabalho docente ao distúrbio de voz.

\section{Conclusão}

Este estudo caso-controle confirmou a associação entre distúrbio de voz em professoras da rede municipal de ensino de São Paulo e estresse no trabalho docente. Foi encontrada diferença estatisticamente significativa entre os grupos de caso e de controle em relação ao estresse no trabalho na condição de alta exigência $(\mathrm{OR}=2,1$; IC95\%: 1,1-3,9), que representa alta demanda associada a baixo controle do trabalho, situação com maior risco de presença de reações adversas à saúde física e mental dos trabalhadores. 


\section{Resumo}

Determinar a associação entre distúrbio de voz e estresse no trabalho em professoras da rede municipal de São Paulo, Brasil é o objetivo deste estudo caso-controle, realizado com professoras com $(n=165)$ e sem $(n=105)$ distúrbio vocal. Foram utilizados os questionários Condição de Produção Vocal $e$ Job Stress Scale. Os grupos são comparáveis nas variáveis de controle, diferenciando-se apenas quanto aos sintomas vocais. Houve diferença estatisticamente significativa entre os grupos em relação ao estresse no trabalho na condição de alta exigência $(O R=2,1$; IC95\%: 1,1-3,9), que representa alta demanda associada a baixo controle do trabalho. Concluindo que a condição de alto desgaste associada ao grupo com distúrbio de voz, neste estudo, é a que apresenta maior risco de adoecimento físico $e$ psíquico ao trabalhador. Ao perder a voz, o professor perde a possibilidade de manter-se em sua função, perde sua identidade profissional, o que coloca, em risco, a sua carreira como educador.

Distúrbios da Voz; Docentes; Saúde do Trabalhador

\section{Referências}

1. Williams NR. Occupational groups at risk of voice disorders: a review of the literature. Occup Med (Lond) 2003; 53:456-60.

2. Vilkman E. Occupational safety and health aspects of voice and speech professions. Folia Phoniatr Logop 2004; 56:220-53.

3. Ferreira LP, Giannini SPP, Figueira S, Silva EE, Karmann DF, Thomé-de-Souza TM. Condições de produção vocal de professores da rede do Município de São Paulo. Distúrb Comun 2003; 14:275-308.

\section{Colaboradores}

S. P. P. Giannini, M. R. D. O. Latorre e L. P. Ferreira contribuíram na concepção do projeto, na análise e interpretação dos dados, na redação do artigo, na revisão crítica relevante do conteúdo intelectual e na aprovação final da versão a ser publicada.
4. Roy N, Merrill RM, Thibeault S, Gray S, Smith EM Voice disorders in teachers and the general population: effects on work performance, attendance, and future career choices. J Speech Lang Hear Res 2004; 44:542-52.

5. Preciado J, Pérez C, Calzada M, Preciado P. Incidencia y prevalencia de los trastornos de la voz en el personal docente de La Rioja. Acta Otorrinolaringol Esp 2005; 56:202-10. 
6. Jardim R, Barreto SM, Assunção AA. Condições de trabalho, qualidade de vida e disfonia entre docentes. Cad Saúde Pública 2007; 23:2439-61.

7. Seligmann-Silva E. Saúde mental e automação: a propósito de um estudo de caso no setor ferroviário. Mental health and automation: remarks on a case study in the railroad industry. Cad Saúde Pública 1997; 13 Suppl 2:95-109.

8. Dejours C. A loucura do trabalho: estudo da psicopatologia do trabalho. 5a Ed. São Paulo: Cortez Editora/Oboré; 2000.

9. Assunção AA, Oliveira DA. Intensificação do trabalho e saúde dos professores. Educação \& Sociedade 2009; 30:349-72.

10. Araújo TM, Graça CC, Araújo E. Estresse ocupacional e saúde: contribuições do modelo demandacontrole. Ciênc Saúde Coletiva 2003; 8:991-1003.

11. Alves MGM, Chor D, Faerstein E, Lopes CS, Werneck GL. Versão resumida da "Job Stress Scale": adaptação para o português. Rev Saúde Pública 2004; 38:164-71.

12. Hirano M. Clinical examination of voice. New York: Springer-Verlag; 1981.

13. Dejonckere P, Remacle M, Freznel-Elbaz E. Reliability and relevance of differentiated perceptua evaluation of pathological voice quality. In: Clemente MP, editor. Voice update. Amsterdam: Elsevier; 1996. p. 321-4.

14. Ferreira LP, Giannini SPP, Latorre MRDO, Zenari MS. Distúrbio da voz relacionado ao trabalho: proposta de um instrumento para avaliação de professores. Distúrb Comun 2007; 19:127-37.

15. Karasek RA. Job demand, job decision latitude and mental strain: implications for job redesign. Adm Sci Q 1979; 24:285-308.

16. Fischer FM, Oliveira DC, Nagai R, Teixeira LR, Lombardi Jr. M, Latorre MRDO, et al. Job control, job demands and health among adolescent workers. Rev Saúde Pública 2005; 39:245-53.

17. Codo W, Menezes IV. Burnout: sofrimento psíquico dos trabalhadores em educação. Cadernos de Saúde do Trabalho 2000; 12:29-34.

18. Araújo TM, Reis EJFB, Carvalho FM, Porto LA, Reis IC, Andrade JM. Fatores associados a alterações vocais em professoras. Cad Saúde Pública 2008; 24:1229-38.

19. Thomé CR. A voz do professor: relação entre distúrbio vocal e fatores psicossociais do trabalho [Dissertação de Mestrado]. São Paulo: Pontifícia Universidade Católica de São Paulo; 2007.
20. Reis EJFB, Araújo, TM, Carvalho FM, Barbalho L, Silva MO. Docência e exaustão emocional. Educação \& Sociedade 2006; 27:251-75.

21. Mizukami MGN. Docência, trajetórias pessoais e desenvolvimento profissional. In: Reali AMMR, Mizukami MGN, organizadores. Formação de professores. São Carlos: EDUFSCar; 1996. p. 59-91.

22. Assunção AA. Saúde e mal-estar do(a) trabalhador(a) docente. In: Anales del VII Seminario Redestrado - Nuevas Regulaciones en América Latina. Buenos Aires: Agencia Nacional de Promoción Científica y Tecnología; 2008. p. 1-20.

23. Esteve JM. O mal-estar docente. Lisboa: Escher/ Fim de Século; 1992.

24. Nóvoa A. A relação escola-sociedade: novas respostas para um velho problema. In: Serbino RV Ribeiro R, Barbosa RLL, Gebran RA, organizadores. Formação de professores. São Paulo: Universidade Estadual Paulista Júlio de Mesquita Filho; 1998. p. 19-39.

25. Giannini SPP, Passos MC. Histórias que fazem sentidos: as determinações das alterações vocais do professor. Distúrb Comun 2006; 18:245-57.

26. Rodrigues S, Azevedo R, Behlau M. Considerações sobre a voz falada. In: Marchesan IQ, Zorzi JLE, Gomes, LCD, organizadores. Tópicos em fonoaudiologia. São Paulo: Lovise; 1996. p. 701-11.

27. Jardim R, Barreto SM, Assunção AA. Voice disorder: case definition and prevalence in teachers. Rev Bras Epidemiol 2007; 10:625-36.

28. Giannini SPP, Ferreira LP, Latorre MRDO. Distúrbio de voz: como definir caso em estudos epidemiológicos? In: Anais do 19o Congresso Brasileiro e 8o Congresso Internacional de Fonoaudiologia. http://www.sbfa.org.br/portal/anais2011/traba lhos_select.php?tt=Busca\&id_artigo=1166 (acessado em 09/Jul/2012).

29. Souza TMT. Saúde vocal no ingresso de professores na Prefeitura Municipal de São Paulo: um programa de promoção da saúde e vigilância. In Anais do 16o Congresso Brasileiro de Fonoaudiologia. http://www.sbfa.org.br/portal/anais2008/ anais_select.php?op=buscaresultado\&cid $=374 \&$ ti d=1 (acessado em 21/Dez/2009).

Recebido em 02/Mar/2012

Versão final reapresentada em 09/Jul/2012 Aprovado em 07/Ago/2012 